\title{
Communication
}

\section{Virtual and Real Water. What is the Difference?}

\author{
Tatiana Yakhno ${ }^{1,2 *}$, Vladimir Yakhno ${ }^{1,2 *}$ \\ 1 Federal Research Center Institute of Applied Physics of the Russian Academy of Sciences (IAP RAS), \\ Nizhny Novgorod 603950, Russia \\ 2 N. I. Lobachevsky State University of Nizhny Novgorod (National Research University), \\ Nizhny Novgorod 603950, Russia \\ * Correspondence: yakhta13@gmail.com (T.Y.); yakhno@appl.sci-nnov.ru (V.Y.); \\ Tel.: (011)-7-831-436-85-80 (T.Y. \& V.Y.)
}

\begin{abstract}
The paper provides information on significant contamination of real laboratory water with hydrophilic microimpurities. This fact suggests that researchers are practically dealing with microdispersed systems. However, this fact is usually neglected in the discussions of the causes of the anomalous properties of water. We will show that, when exposed to various factors of physical nature, water demonstrates reactions of the same type, namely, increased $\mathrm{pH}$ and electrical conductivity, reduced redox potential and viscosity, and enhanced bioavailability. Each exposure is accompanied by the destruction of aggregates of the dispersed phase and its transition to a fine-dispersed state. The relationship between this phenomenon and the change in the physicochemical properties of water is discussed.
\end{abstract}

Keywords: water structuring; exclusion zone; interfacial water; hydrophilic surface; biological effects; high dilution effects

\section{Introduction}

It has long been supposed that purified laboratory water is freed from impurities and becomes just a mixture of $\mathrm{H}_{2} \mathrm{O}$ molecules capable of combining into one or another molecular cluster with femtosecond relaxation times. This information formed the basis for molecular modeling and explanation of the known thermodynamic anomalies of water [1-7]. Moreover, as a rule, it was not taken into account that real water always contains an impurity microdispersed phase [8] which is a full participant of the dynamic processes in this open nonequilibrium system.

According to the data of the National Institutions of Health [9], "Water is easily contaminated by chemical solids, gases, vapors and ions that leach from conduit lines and containers. These may include sodium and silica from glass, plasticizers and ions from piping, microbial species and their endotoxins, as well as particulate contaminants (Millipore, n.d.). Soluble organic contaminants can even be introduced from deionizer resins used in the treatment process, especially if inadequate resins are selected or resins were previously contaminated." Any method of water purification distillation, filtration and ultrafiltration, reverse osmosis and deionization - has its own drawbacks. In particular, deionized water is often called "hungry water" which is easily contaminated and can cause corrosion of many materials. Even electrodeionization does not remove organics, particles, pyrogens and bacteria, although water may be less susceptible to microbial contamination than layers of ion-exchange resins [9].

The authors of [10] stated that the control parameters of "pure" (distilled, twice and thrice distilled, deionized) water are determined by the manufacturers in closed vessels, before it comes into contact with air. Less than a minute after the water has been removed from the container and contacted air, it turns into a weak solution of carbonic acid due to the fast dissolution in it of carbon dioxide. Thorough cleaning of the elements of the experimental setup is also obligatory in experiments with water. The experiments should be carried out in a dust and odor free room. 
In our earlier works $[8,11]$ we mentioned the microstructures detected by different authors in distilled, bidistilled, and deionized water using various physical methods of research. Here we will show that these microstructures can be observed in a conventional light microscope, and their size and concentration correspond to the mass of dry residue after evaporation of free water. It is known that, when hydrophilic particles get into water, they interact with it acquiring a hydration shell at the interface - a new low-entropy phase of water. According to the present-day concepts, this phase the Exclusion Zone (EZ) [12] - has a quasi-ice structure capable of displacing from this zone all ions and microimpurities in the course of its growth. This leads to an increase in the osmotic pressure in bulk water (disperse medium). EZ is sensitive to osmotic pressure and, starting from a certain threshold, begins to "melt", turning into free water [8]. As a result, the osmotic pressure in bulk water decreases, and the EZ again starts to grow around hydrophilic microparticles [13]. These events underlie the slow self-oscillatory processes observed in colloidal liquids [14]. We believe that the microimpurities contained in water, as well as their hydration shells play an important role both in determining a number of its physical properties and in organizing dynamic processes in liquids. In the present communication, we will consider this issue on specific examples.

\section{Results and Discussion}

As mentioned above, microstructures in water and aqueous solutions can be easily detected using a conventional light microscope. Figure 1 shows the microstructures present in the liquid phase of the studied media. One can see that their size and concentration correspond to the relative dry mass of these liquids.

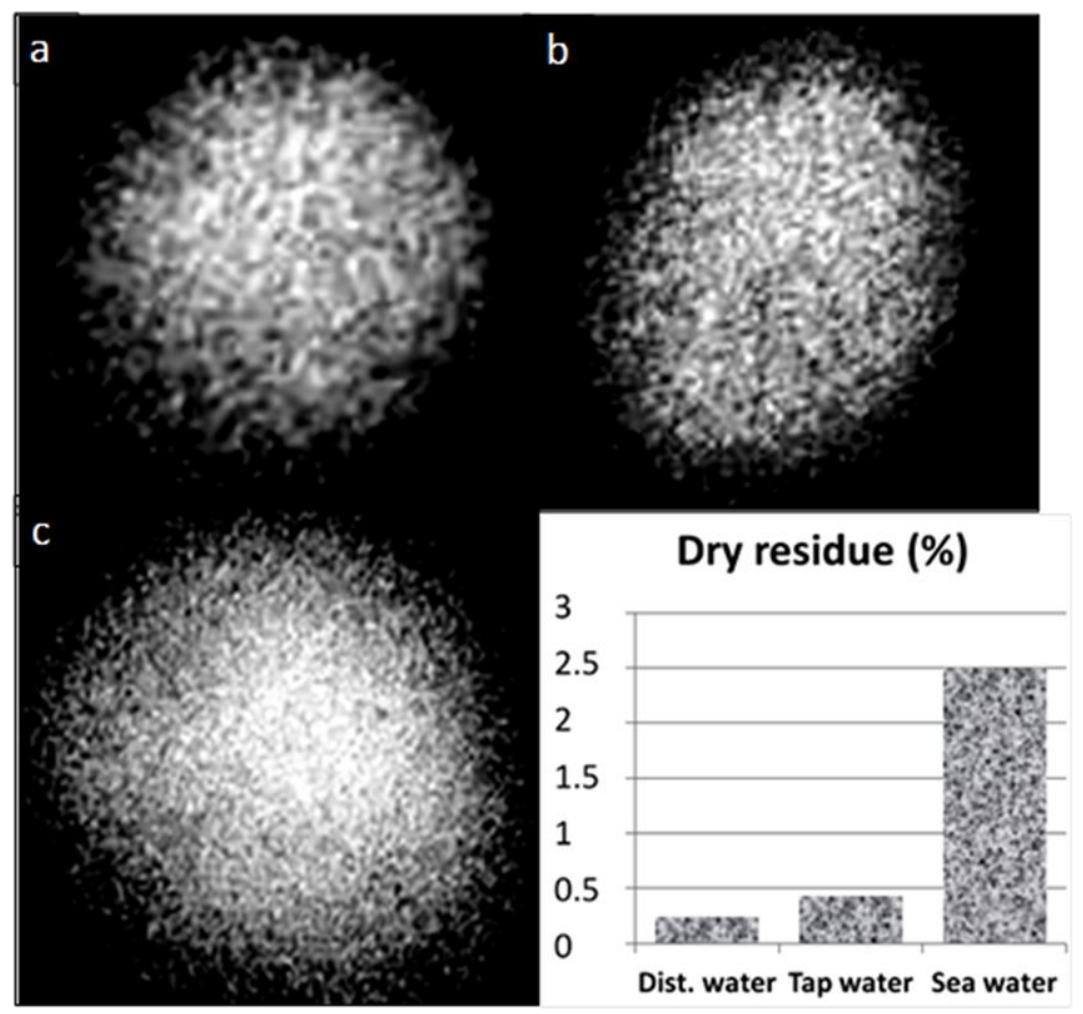

Figure 1. The microphase of aqueous media in the "hanging drop" preparation placed into a hole in a plastic plate. The width of each frame is $0.5 \mathrm{~mm}$ : a - distilled water, $\mathrm{b}$ - tap water, $\mathrm{c}-$ water of the Black Sea. The diagram shows the relative content of solids in each sample after evaporation of free water.

The phenomenon of increasing electrical conductivity of water and aqueous solutions during their storage in a closed glass container was studied in the work [15]. A significant increase in the electrical conductivity of aged solutions (hundreds of days) compared to that of chemically similar one-day-old solutions was detected at $25^{\circ} \mathrm{C}$ and $5^{\circ} \mathrm{C}$ in all cases, except for the solutions frozen at 
$-20^{\circ} \mathrm{C}$. The authors hypothesized that hydrophilic surfaces can affect the increase in electrical conductivity of the liquids in contact with them. In our opinion, hydrophilic microimpurities contained in water should also be included in the concept of "hydrophilic surfaces".

In our experiments we observed mainly sodium chloride microcrystals surrounded by EZ-like hydration shells $[8,11]$, which are also able to actively influence the conductivity parameters. In addition, the microdispersed phase in water forms large-scale (hundreds of microns) aggregates according to the Gibbs law - the tendency of the system to reduce its free energy by reducing the total surface area:

$$
\sigma \mathrm{S} \rightarrow \mathrm{min}
$$

where $\sigma$ is the specific value of the free energy of the surface and $S$ is the total surface area of the dispersed phase.

If $\sigma$ is constant, there occur spontaneous processes reducing the system dispersion, thereby resulting in a decrease in the total surface area $(S)$ and energy release (the Gibbs - Helmholtz equation [16]):

$$
\mathrm{Us}=\sigma+\mathrm{qs}=\sigma-\mathrm{T}(\mathrm{d} \sigma / \mathrm{dT}) \mathrm{p},
$$

where Us is the total surface energy of the system, $\sigma$ is the surface tension, and qs is the heat of formation of a unit of surface area.

In lyophilic systems, the energy of coagulation bonds is not high (comparable with the energy of Brownian motion kT); therefore, a high-energy impact is not needed to disperse the aggregates. Let us consider a number of experimental facts, which, from our point of view, can be related to the degree of dispersion of microstructures (aggregated or disaggregated state).

Data on the interesting phenomenon - the change in a number of physical characteristics of water heated to $50-60^{\circ} \mathrm{C}$ - were reported in [17]. The authors of this work inspected the existing literature with the aim to investigate the temperature dependence of a variety of physical properties of water, including thermal conductivity, refractive index, conductivity, and surface tension.

According to our observations, when water is heated, large aggregates of microstructures are destroyed and the suspension becomes more homogeneous (Figure 2).
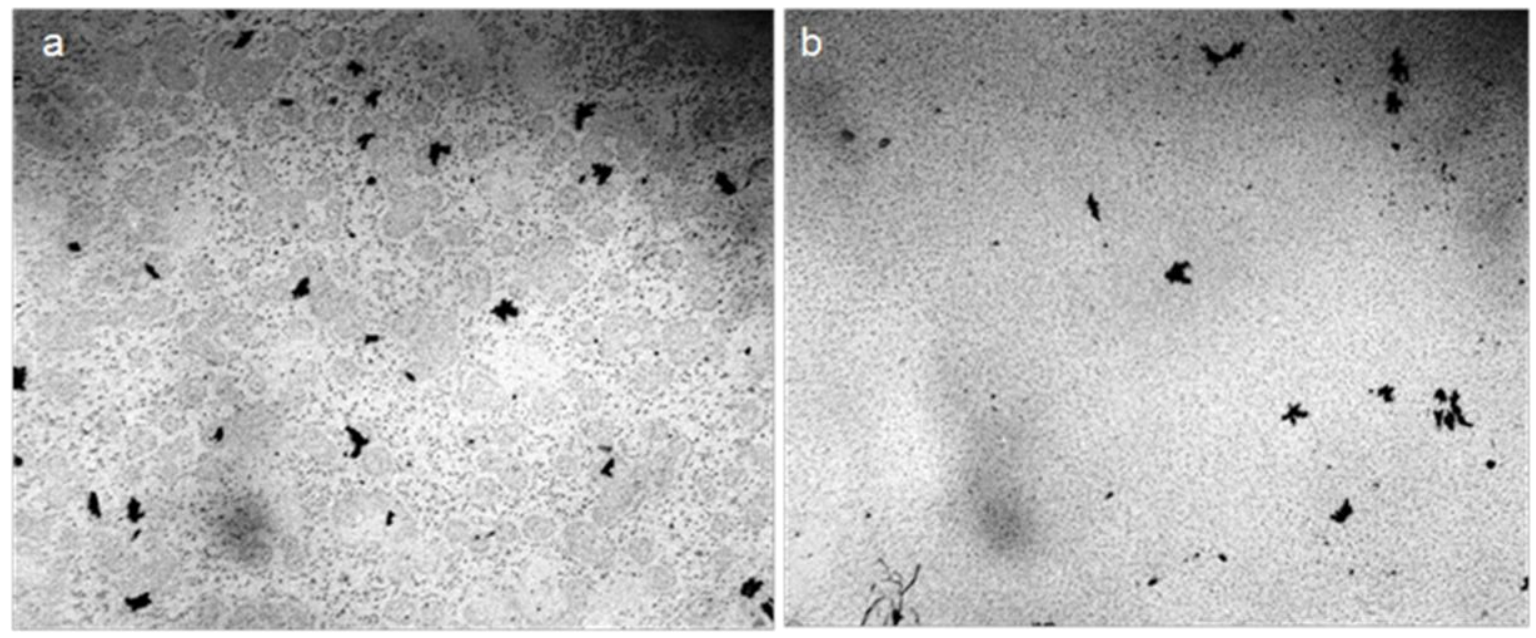

Figure 2. Microscopy of distilled water between the slide and cover glasses. The thickness of the water layer is $16 \mu \mathrm{m}$, the width of each frame is $3 \mathrm{~mm}$ : a - initial sample before boiling; $b$ - water after heating to boiling temperature in a microwave oven and subsequent cooling to room temperature. The black fractal clusters in the images are hydrophobic microimpurities which are not discussed in this communication. 
In our earlier work we showed that the process of water boiling is accompanied by the qualitative changes in its physicochemical properties like those under the action of hydroimpact-cavitation for 1.5 hours, including degassing and an increase in $\mathrm{pH}$, electrical conductivity, and ultrasound speed [18]. The observed changes relax with time (up to 3 days). We believe that the change in a number of physical parameters considered in this work may be a consequence of the energy impact on water accompanied by degassing and breakdown of its microaggregate structure resulting in an increase of the area of the interface between free and bound water. Since the EZ surface has a negative potential, the number of "seats" for protons in the liquid increases significantly, which leads to alkalization of the solution.

Irradiation by low-intensity light of a semiconductor laser pointer makes an analogous impact on the aggregation state of water (Fig. 3). A decrease in the electric capacity of the liquid by $\sim 1 \mathrm{pf}$ was noticed in this case [19].
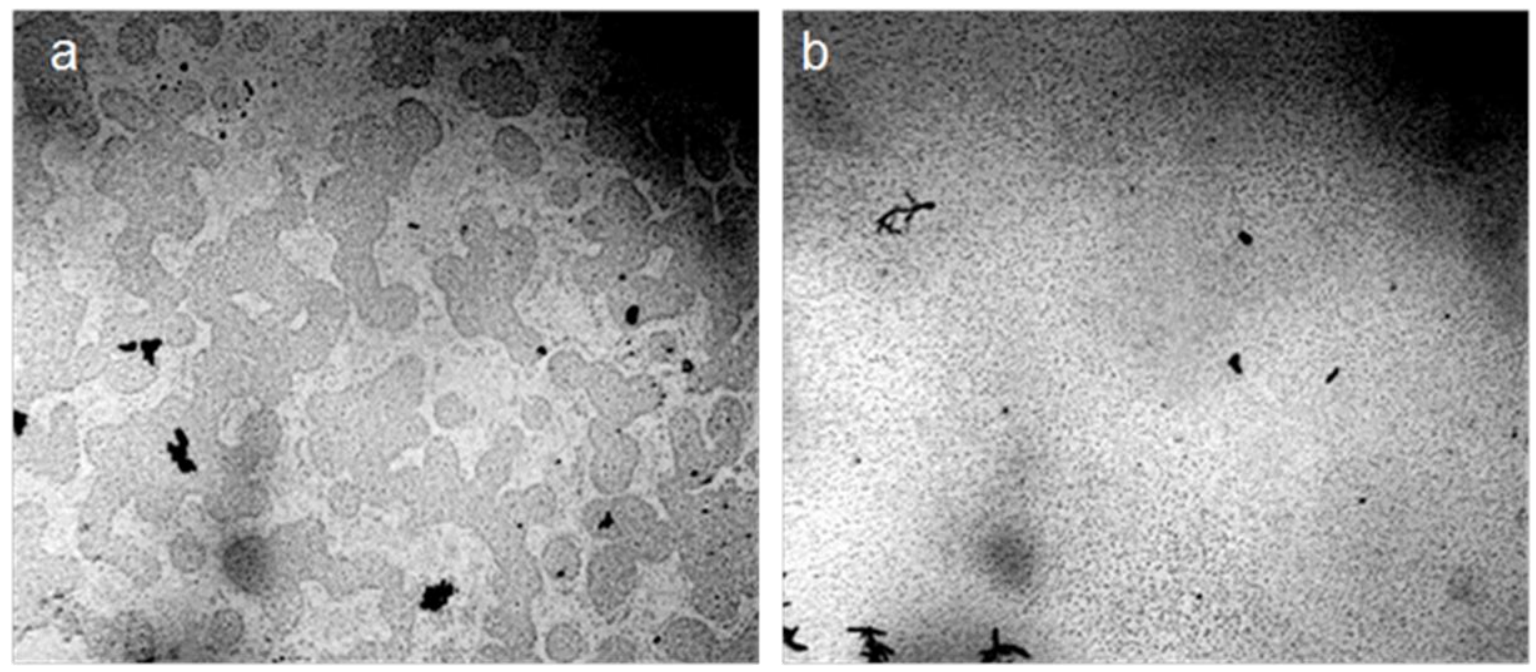

Figure 3. The microstructure of mineral natural drinking water "Seraphim Dar." Microscopy of a liquid layer with a thickness of $\sim 16 \mu \mathrm{m}$ before (a) and after (b) irradiation of a glass of water with a $20 \mathrm{~mW}$ green laser for 10 minutes.

The review of the literature on the results of water exposure to different disturbing factors of physical nature showed identical consequences - an increase in $\mathrm{pH}$, a decrease in redox potential, an increase in electrical conductivity, and a decrease in viscosity. All this taken together enhances the bioavailability of water for plants and animals. We will illustrate this statement with concrete examples. In the work [20] bidistilled water was exposed to strong spatially inhomogeneous fields. As a result, it became a better solvent than distilled water, while it had lower viscosity, lower surface tension and a higher wetting coefficient. It was shown that such modified water acquired increased biological activity. The authors of that work associated the mechanism of such changes with additional dissociation of water molecules and with the formation of protonophilic $\mathrm{H}+\left(\mathrm{H}_{2} \mathrm{O}\right) \mathrm{p}$ and negative $\mathrm{OH}-\left(\mathrm{H}_{2} \mathrm{O}\right) \mathrm{n}$ cluster ions. Basically the same changes (increased $\mathrm{pH}$, reduced redox potential and increased electrical conductivity) occur in water after cavitation [21]. It was shown that after treatment all the measured parameters of the activated water were not restored for $200 \mathrm{~min}$. Changes in the physicochemical properties of deionized water as a result of electromagnetic exposure and various degassing procedures were assessed in [22]. It was found that, independent of the method of acting on deionized water, its electric conductivity and $\mathrm{pH}$ increased and redox potential decreased. Degassing by evacuation and blowing with inert gas gave almost the same result. The changes in the water properties were the most pronounced when it was exposed to a high-frequency electromagnetic field. The influence of the physical fields on the physicochemical properties of water was studied in [23]. The impact of He-Ne laser radiation, constant magnetic field, and ultrasonic radiation on the $\mathrm{pH}$ and electrical conductivity of tap water was investigated. A small increase in $\mathrm{pH}(3.8 \%-5.8 \%)$ was observed for all 3 types of physical action on water. Ultrasound affected the water $\mathrm{pH}$ most of all [23]. The increase in the $\mathrm{pH}$ of the control water sample was 
associated with water degassing with time. On exposure to laser radiation, the electrical conductivity of water increased by $21 \%$. Under the action of ultrasonic radiation the electrical conductivity of water increased by $46 \%$. Under the influence of a magnetic field, the electrical conductivity of water changed only slightly (4\%). It was found [24] that the water pre-activated by a magnetic vector potential changed the mobility of the introduced infusoria, yeast fermentation and the preferred consumption by mice. It was also shown that some physical parameters such as the UV absorption spectrum and the state of silica admixture in water changed after its activation. It was suggested that, when biological objects are exposed to many weak electromagnetic fields, water is the primary target. The increase in $\mathrm{pH}$ and electrical conductivity of distilled water after exposure to a constant magnetic field, laser radiation and ultrasound for 5-20 minutes was attributed by the authors of [25] to a change in the structure of water, an increase in the number of charged particles, their mobility and activity.

It was interesting to see how things stand with melt water recommended for a healthy diet. Doctors note that fresh melt water helps accelerate recovery processes, increases body's resistance to infections, reduces the sensitivity of the mucous membrane, and normalizes the tone of bronchial muscles [26]. We examined the microstructure of tap water before and after freezing - thawing (Fig. 4). We found that it loses part of the microdispersed phase which is detached during defrosting in the form of loose flakes, whereas the melted snow looks like a colloidal crystal due to the regular arrangement of the microstructures on the glass surface (Figure 5).
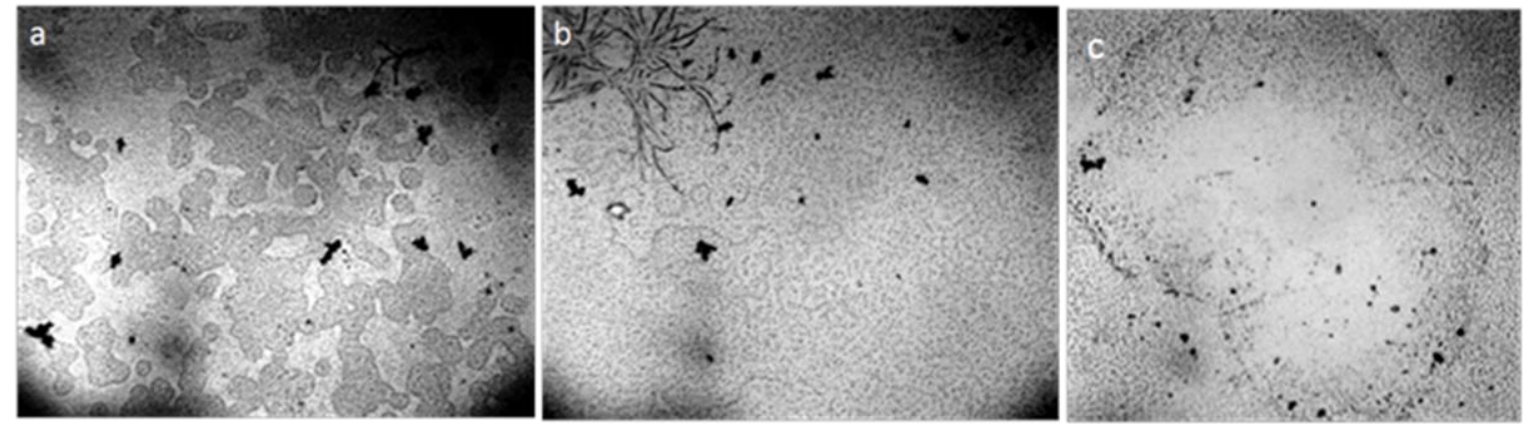

Figure 4. Microscopy of a thin layer $(\sim 8 \mu \mathrm{m})$ of distilled water before freezing (a) and after freezing and thawing (b); a clot of dispersed phase in thawed water (c). The width of each frame is $3 \mathrm{~mm}$.

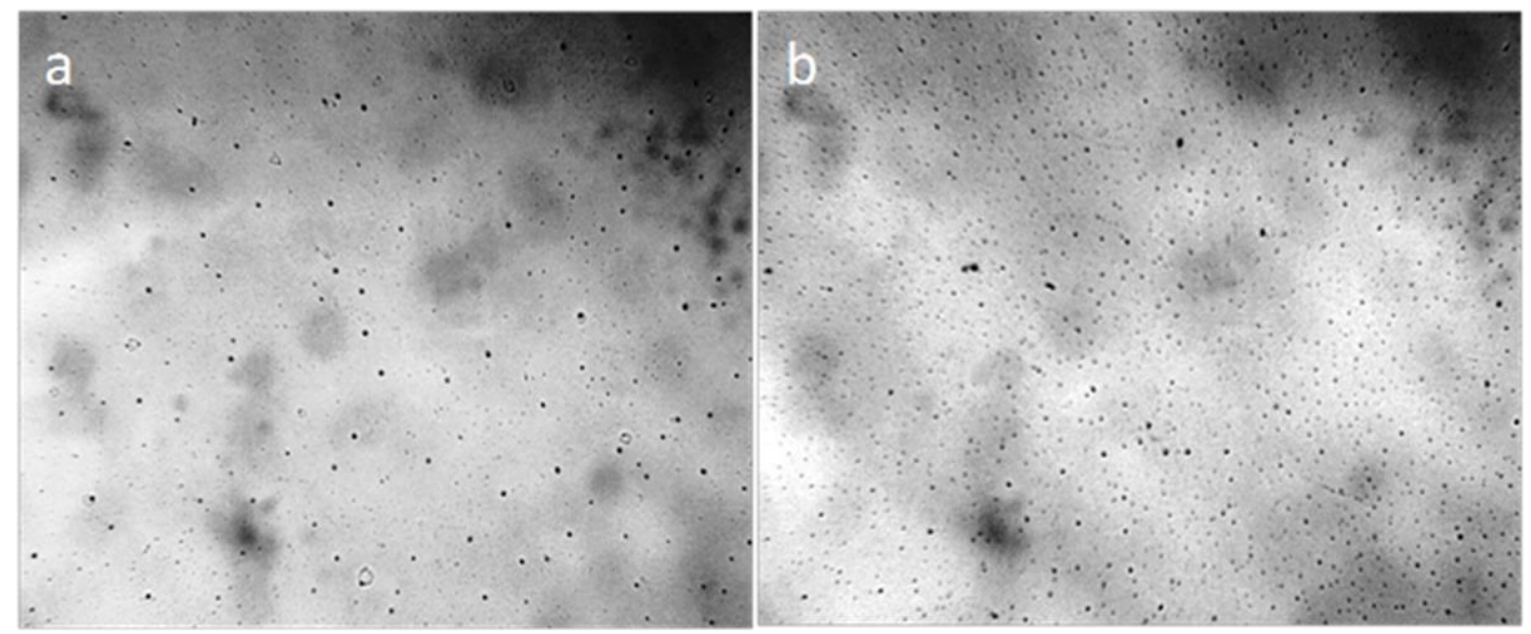

Figure 5. Melted snow (a, b - different fields of view) in a thin layer $(\sim 8 \mu \mathrm{m})$ between the slide and cover glasses. The width of each frame is $3 \mathrm{~mm}$.

Bearing in mind that, in contrast to virtual water, real water contains an additional, microdispersed phase, we believe that this fact should not be ignored when considering the physicochemical properties of water. In particular, the true reason for the regular changes in the properties of water and the increase in its biological activity can be largely attributed to the 
dispersion of aggregates of hydrated impurities or (in the case of melt water) with the removal of a significant part of impurities from liquid water. The presented results also show that for the water to be "activated" by physical methods it is sufficient to boil it or whip it with an electric mixer (that's just what we did in a cup in our kitchen). We also believe that our observations will be useful for studying the "ultrahigh dilution effect" accompanied by periodic vigorous shaking of liquid media.

\section{Materials and Methods}

The experiments were carried out under laboratory conditions at $\mathrm{T}=21-23^{\circ} \mathrm{C}, \mathrm{H}=67-70 \%$. Samples of distilled water (TU 2384-009-48326337-2015, specific conductivity $4.5 \mu \mathrm{S} / \mathrm{cm}, \mathrm{pH} 7.0$ ), tap water (specific conductivity $550 \mu \mathrm{S} / \mathrm{cm}$ ), and mineral natural drinking water "Seraphim Dar" (mineralization $0.05-0.12 \mathrm{~g} / \mathrm{L}$ ) were used for microscopic observations. In addition, the water of the Black Sea was studied (salinity $\sim 3.5 \%$ ). The structure of the water placed in the hole $3-\mathrm{mm}$ in diameter in a plastic plate was examined. The dry mass of the water samples was determined gravimetrically (torsion balance up to $500 \mathrm{mg}$ ). The studies were performed under a Levenhuk microscope with a video camera coupled to a computer using the ToupView program. The microscope slides and cover glasses produced by ApexLab (Russia) with dimensions $25.4 \mathrm{~mm} \times 72.2$ $\mathrm{mm} \times 1 \mathrm{~mm}$ and $24 \mathrm{~mm} \times 24 \mathrm{~mm} \times 0.6 \mathrm{~mm}$, respectively, and Petri dishes $\mathrm{d}=35 \mathrm{~mm}$ (polystyrene, sterile, MiniMed, Russia) were used. We took only new glass and plastic dishes without additional treatment. Samples of liquids in the form of droplets in a volume of $5 \mu \mathrm{L}$ or $10 \mu \mathrm{L}$ with a Sartorius microdoser (Biohit) were applied onto the substrates and covered with a coverslip. The water was examined under a microscope as a thin layer between the slide and cover glasses before and after freezing in the Petri dishes in the chamber of the domestic cooler $\left(\mathrm{T}=-20^{\circ} \mathrm{C}\right)$. Natural snow taken from the depths of the snowdrift was also used. The surface of mineral water poured into a $50 \mathrm{ml}$ glass beaker was irradiated with light of a $20 \mathrm{~mW}$ semiconductor laser pointer for 10 minutes under laboratory conditions. The distance between the light source and the surface of the water did not exceed $1 \mathrm{~cm}$. The microstructure of water in a thin layer was studied before and after irradiation.

\section{Conclusion}

In this communication, we have just outlined the problem of differences between real and virtual water and put forth related arguments in favor of our ideas. The presence of the microdispersed phase in real water radically changes its physicochemical properties and allows one to explain both a number of known water anomalies and the uniformity of its reaction to various disturbing influences. We hope that it will be interesting for the scientific community and worthy of further studies and discussion.

Author Contributions: Conceptualization, T.Y. and V.Y.; methodology, T.Y.; validation, T.Y., V.Y; investigation, T.Y., V.Y.; writing - original draft preparation, T.Y.; funding acquisition, V.Y.

Funding: This project was funded by the Ministry of Education and Science of Russia (project No 14.Y26.31.0022).

Acknowledgments: The authors are deeply grateful to their colleagues A. Sanin and A. Pakhomov for technical support.

Conflicts of Interest: The authors declare no conflict of interest.

\section{References}

1. Malenkov, G.G. Structure and dynamics of liquid water. J. Struct. Chem. 2006, 47, S5-S35.

2. Sarkisov, G.N. Structural models of water. Phys. Usp. 2006, 49, 809-820.

3. Nilsson, A.; Pettersson, L.G.M. Perspective on the structure of liquid water. Chem. Phys. 2011, 389, 1-34.

4. Mel'nichenko, N.A. Structure and dynamical properties of liquid water. Vestn. Far East Branch Russ. Acad. Sci. 2010, 1, 65-74 (In Russian).

5. Maestro, L.M.; Marqués, M.I.; Camarillo, E.; Jaque, D.; García Solé, J.; Gonzalo, J.A.; Jaque, F.; del Valle, J.C.; Mallamace, F.; Stanley, H.E. On the existence of two states in liquid water: Impact on biological and nanoscopic systems. Int. J. Nanotechnol. 2016, 13, 667-677. 
6. Soper, A.K. Structures of high-density and low-density water. Physical Review Letters, 2000, 84, 13, 28812884.

7. Nilsson, A.; Pettersson, L.G.M. Perspective on the structure of liquid water. Chem. Phys. 2011, 389, 1-34.

8. Yakhno, T.; Yakhno, V. A study of structural organization of water and aqueous solutions by means of optical microscopy. Crystals 2019, 9, 52.

9. Laboratory Water. Its Importance and Application. National Institutions of Health, 2013, 22 p. https://www.orf.od.nih.gov/TechnicalResources/Documents/DTR\%20White\%20Papers/Laboratory\%20W ater-Its\%20Importance\%20and\%20Application-March-2013 508.pdf

10. Karakashev, S.I.; Firouzi, M.; Wang, J.; Alexandrova, L.; Nguyen, A.V. On the stability of thin films of pure water. Advances in Colloid and Interface Science 2019, 268 82-90. https://doi.org/10.1016/j.cis.2019.03.005

11. Yakhno, T.; Drozdov, M.; Yakhno, V. Giant water clusters: Where are they from? Int. J. Mol. Sci. 2019, 20, 1582; doi:10.3390/ijms20071582 : https://www.mdpi.com/1422-0067/20/7/1582/pdf

12. Pollack, G. The Fourth Phase of Water: Beyond Solid, Liquid and Vapor; Ebner \& Sons: Seattle, WA, USA, 2013; 357p.

13. Yakhno, T.A.; Yakhno, V.G. Water-induced self-oscillatory processes in colloidal systems by the example of instant coffee. J. Basic Appl. Res. Int. 2017, 20, 70-83.

14. Yakhno, T.A.; Yakhno, V.G. The coffee-drop phenomenon and its time fluctuations: Self-sustained oscillations in colloidal liquids. Tech. Phys. 2017, 62, 347-354.

15. Verdel, N.; Jerman, I.; Krasovec, R.; Bukovec, P.; Zupancic, M. Possible time-dependent effect of ions and hydrophilic surfaces on the electrical conductivity of aqueous solutions. Int. J. Mol. Sci. 2012, 13, 4048-4068; doi:10.3390/ijms13044048.

16. Basic Concepts and Equations of Colloid Chemistry, Eds. K.I. Grodsky, N.N. Kienskoy, V.V. Gavrilova, V.V. Nazarova. M .: Mendeleev RCTU, 2013, 40 p.

17. Maestro, L.M.; Marqués, M.I.; Camarillo, E.; Jaque, D.; García Solé, J.; Gonzalo, J.A.; Jaque, F.; del Valle, J.C.; Mallamace, F.; Stanley, H.E. On the existence of two states in liquid water: Impact on biological and nanoscopic systems. Int. J. Nanotechnol. 2016, 13, 667-677.

18. Yakhno, T.A.; Uvarov, V.M.; Sanin, A.G.; Kazakov, V.V. Hydro-shock-cavitation effect on water, or where do protons disappear? Materials of reports of the V Congress of Biophysicists of Russia, October 4-11, Rostov-on-Don, 2015, 2, 339 (in Russian).

19. Yakhno, T.A.; Pakhomov, A.M.; Sanin, A.G.; Yakhno, V.G. The effect of low-intensity light on the structural and dynamic parameters of water. Scientific works of the VIII International Congress "Weak and Super-Weak Fields and Radiations in Biology and Medicine", 8,.99, St. Petersburg, 2018 (in Russian) www.biophys.ru/archive/congress-2018.pdf\#page=99

20. Gulyaev, Yu.V.; Eremin, S.M.; Markov, I.A.; Novoselova, E.G.; Novikov, V.V.; Ten, Yu.A.; E.E. Fesenko. Physicochemical properties of reagent-free water and its biological activity. Journal of Radio Electronics, 2005, 11 (electronic journal, ISSN 1684-1719) (in Russian).

21. Kulagina, V.A.; Sapozhnikova, E.S.; Stebeleva, O.P.; Kashkina, L.V.; Zheng, Zhi-Ying; Li, Qian; Li, Feng-Chen. Features of the effect of cavitation effects on the physicochemical properties of water and effluents. Journal of Siberian Federal University. Engineering \& Technologies, 2014, 5, 7, 605-614 (in Russian).

22. Chirkova, V.Yu.; Stas', I.E. Assessment of changes in the physicochemical properties of deionized water as a result of electromagnetic exposure and various degassing methods. DOI 10.14258 / izvestia.asu.ru, 2014, 31-44 (in Russian).

23. Musienko, K.S.; Ignatova, T.M.; Glazkova, V.V. The study of the influence of physical fields on the physicochemical properties of water. Biomedical Engineering and Electronics, 2014, 2. URL:.esrae.ru/199-963 (in Russian).

24. Anosov, A.V.; Trukhan, E.M. The variation of the vector potential in the laboratory changes the biological properties of water. Biophyzika, 2012, 57, 3, 389-394 (in Russian).

25. Bukaty, V.I.; Nesteryuk, P.I. Measurement of physical-chemical characteristics of water under various physical influences, taking into account transient processes. Polzunovsky vestnik, 2010, 2, 60-65 (in Russian).

26. Internet resource. Available online: https://aif.ru/health/food/1446590 (accessed on 16.02.2015). 